\title{
@OPENACCESS COMPARISON OF HYPERTENSION, ITS CONTROL AND check tor updates COMPLIANCE OF ANTI-HYPERTENSIVES AMONG DEPRESSED AND NON-DEPRESSED PATIENTS
}

\begin{abstract}
${ }^{1}$ Department of Cardiology, Lady Reading Hospital (Medical Teaching Institute), Peshawar-Pakistan.

2 Category-D Hospital, Health Department, Government of Khyber Pakhtunkhwa, Peshawar-Pakistan
\end{abstract}

Address for correspondence: Ayesha Khalil

Department of Cardiology, Lady Reading Hospital (Medical Teaching Institute), Peshawar-Pakistan.

E-mail:

ayesha70khalil@gmail.com

Date Received:

February, 22 $2^{\text {nd }} 2021$

Date Revised:

May, $21^{\text {st }} 2021$

Date Accepted:

June, $21^{\text {st }} 2021$
This article may be cited as Iqbal MA, Khalil A, Subhan S, Khan M, Gul A. Comparison of hypertension, its control and compliance of anti-hypertensives among depressed and non-depressed patients. J Postgrad Med Inst 2021;35(2):96-9. https://doi.org/10.54079/ jpmi.35.2.2855.

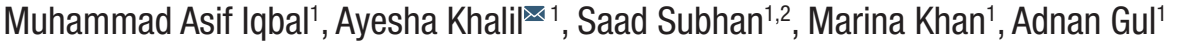

ABSTRACT

Objective: To compare frequency of hypertension, control of blood pressure and compliance to anti-hypertensives among depressed versus non-depressed patients.

Methodology: This cross-sectional study was conducted in the Department of Cardiology Lady Reading Hospital, Peshawar. The patients were interviewed for the diagnosis of depression and compliance to anti-hypertensives. PHQ 9 questionnaire was used to diagnose depression among study patients. Total patients were 1026 divided into two groups, group A were 634 patients and group B were 392 patients. Group A were patients having moderate, moderately severe and severe depression and Group B patients were having no depression and mild depression. The two groups were compared for the frequency of hypertension, and compliance to drugs using Morisky medication adherence scale. SPSS v.20.0 was used to analyze the data.

Results: The mean age of the sample was $55.86 \pm 11.37$ years with male to female ration of 1.5:1. Hypertension was present in $69.24 \%$ of patients in group $A$ and $60.46 \%$ group $B(p=0.006)$. Mean SBP was $131.17+24.7$ $\mathrm{mmHg}$ in depressed patients and $124.20+19.7 \mathrm{mmHg}$ in non-depressed $(p=0.000)$. Similarly mean DBP was $82.06+13.3 \mathrm{mmHg}$ in depressed and $78.99+12.1 \mathrm{mmHg}$ in non-depressed patients $(p=0.004) .335 / 392$ non-depressed patients were compliant to medication as compared to 512/634 depressed patients $(p=0.052)$.

Conclusion: Hypertension was found to be more prevalent among depressed patients. In compliance to anti-hypertensives, depressed patients had lesser compliance than the patients of the other group.

Key Words: Hypertension; Depression; Patient's Compliance.

\section{INTRODUCTION}

Depression is a mental health disorder in which patients have low mood and energy. This in turn effects person's feelings, thoughts, behavior and overall sense of well-being. Depressed patients usually exhibit feeling of insomnia, loss of appetite, having easy fatigability, irritability, have problems in decision making and having suicidal thoughts. ${ }^{1}$ Depression is estimated to affect 350 million people around the world with a lifetime risk of $7 \% .{ }^{2}$ It is common in patients suffering from chronic illness including Diabetes, hypertension and Heart diseases. ${ }^{1}$ Hypertension is the most common disease worldwide. Globally an estimated $26 \%$ of the world's population (972 million people) has hypertension and its prevalence is expected to increase to $29 \%$ by 2025. As Hypertension causes and complicates many diseases including Heart Attack, heart failure and stroke, it is the leading causes of death worldwide. High blood pressure is the most common modifiable risk factor for Coronary artery disease(CAD) and cerebrovascular accidents (CVA). ${ }^{3,4}$ Depression is commonly associated with hypertension and may interfere with blood pressure control. ${ }^{5}$

A study highlighted three times higher frequency of depressive symptoms in hypertensive patients. There is need for reassurance and psychological feedback in hypertensive patients in order to improve control and compliance of blood pressure in such patients. ${ }^{6} \mathrm{An}$ increased prevalence of hypertension in depressed patients has been described by Adamis et al ${ }^{6}$ in their study of correlation between psychiatric illness and physical diseases in 75 elderly patients. They observed that depressed patients had more cardiovascular diseases and hypertension than any other psychiatric patients. Rabkin et al found that frequency of major depression was three times higher in patients being treated for hypertension. ${ }^{5}$ Depression not only led to hypertension, the opposite is also true as Hypertension may cause 
depression by inducing cerebrovascular and ischemic changes in the brain, predisposes individuals with hypertension to depression. ${ }^{7}$

Despite high prevalence and impact of depression and as a contributor of other diseases, it is usually not detected by primary care physicians nor receives adequate treatment. Unrecognized and untreated depression, not only affects quality of life but may also interfere with the treatment and prognosis of other chronic diseases such as ischemic heart disease. ${ }^{8,9}$ It is not clear from the existing state of knowledge that depression affects hypertension negatively and how much is the control of blood pressure and compliance among depressed patients in our local study. Even modest improvements in hypertensive patients may have a significant impact on the compliance and control of the whole population. ${ }^{9}$

The aim of this study was to determine the effect of depression in terms of frequency of hypertension among depressed patients, control of systolic and diastolic Blood Pressure and compliance to drugs in our local population. Rationale of the study is that by determining the true magnitude of the disease, treating the depressed patients can improve compliance and control of hypertension, thereby improving management and outcome of hypertension.

\section{METHODOLOGY}

For this cross-sectional study conducted at Department of Cardiology, Lady Reading Hospital Peshawar, sample size was calculated using online calculator http://www.calculator.net, keeping 95\% confidence level, $5 \%$ margin of error and $26.8 \%$ frequency of hypertension among depressed patients, as reported by Zhanzhan Li. $^{10}$ After taking the ethical approval from the hospital, the sample was recruited using non-probability convenient sampling. Patients presenting to OPD or ward of Cardiology Department were assessed for enrolment. Patients of age 18 years or above who came to Cardiology OPD or admitted to cardiology ward were enrolled in the study. Their informed consent was taken. Exclusion criteria for study patients was unconscious patients or who cannot give proper history, patients having other associated psychotic features, and patients having renal impairment. This study was conducted from March 2018 to March 2019. PHQ-9 scale was used to diagnose depression. Study patients were categorized into two Groups based on PHQ-9 scale. Group A were patients having mild, moderate, moderately severe and severe depression and Group B patients having no or minimal depression. Both group were similar to each other in other parameters like age, gender, socio economic status and educational background.

PHQ-9 scale categorize depression into 5 categories based on the score patient achieve using this scale. Categories are (1) no depression (score: 0-4), (2) mild depression (score: 5-9), (3) moderate depression (score: 10-14), (4) moderately severe (score: 15-19) and (5) severe depression (score: 20-26).

Patients were interviewed for the diagnosis of depression and compliance to drugs. Past and present history of the patients were recorded, clinical examination performed, blood pressure checked and values recorded. Investigations such as Renal Functions Tests for exclusion criteria was done. Patients having history of Hypertension, using antihypertensive medications or having systolic blood pressure (SBP) more than $140 \mathrm{~mm} \mathrm{Hg}$ and/ or Diastolic Blood pressure (DBP) more than $90 \mathrm{mmHg}$ on two occasions, were considered as Hypertensive. The two groups were compared for the presence of hypertension and mean systolic and diastolic Blood Pressure and compliance to drugs. Compliance to drugs was checked using Morisky scale. Patients having high and moderate adherence on the basis of Morisky scale were taken as having compliance to drugs whereas those patients having low adherence on the basis of Morisky scale were taken as no compliance to drugs. Morisky sub group analysis as High adherence, Medium adherence and low adherence was determined for both groups in sub group analysis using Morisky score as $>8,8-6$ and $<6$ as high medium and low score respectively. Data was entered on a pre- determined proforma.

Data was analyzed using statistical package for social sciences (SPSS) 20. Qualitative values were presented in percentage. Quantitative values were presented in the form of mean $\pm S D$. P-value less than 0.005 was considered statistically significant.

\section{RESULTS}

There were a total of 1026 patients in the study. Out of total, Group A was having 634 patients and group B, 392 patients. Male were 631 (61.5\%) and female 395 (38.5\%). Mean age was 55.86 (18-95) years.

Frequency of Hypertension in depressed patients was 439/634 whereas in non-depressed patients it was 238/392 (69.2\% vs $60.7 \%, p=0.005)$. Frequency of hypertension was linearly related with level of depression. Higher level of depression was associated with higher frequency of hypertension as shown in Table-1. Mean SBP was $131.17 \mathrm{mmHg}$ in depressed patients and $124.20 \mathrm{mmHg}$ in non-depressed ( $P$ value (0.000). Similarly mean DBP was 82.06 $\mathrm{mmHg}$ in depressed patients and $78.99 \mathrm{~mm}$ $\mathrm{Hg}$ in non-depressed patients $(p=0.004)$. Compliance among Non-depressed patients is $335 / 392$ as compared to depressed patients $512 / 634$ ( $85 \%$ vs $80 \% p=0.052$ ). In sub group analysis, High compliance was observe in non-depressed group and low in depressed patients as determined by Morisky Scale. The details are given in table 2. 
Table 1: Frequency of Hypertension with level of depression

\begin{tabular}{|c|c|c|c|}
\hline Depression level & Hypertension (\%) & No Hypertension (\%) & P value \\
\hline Mild & $223(55.7 \%)$ & $116(34.2 \%)$ & .000 \\
\hline Moderate & $161(72.5 \%)$ & $61(27.4 \%)$ & .003 \\
\hline Moderate to severe & $45(75 \%)$ & $15(25 \%)$ & .004 \\
\hline Severe & $14(87.5 \%)$ & $2(12.5 \%)$ & .032 \\
\hline
\end{tabular}

Table 2: Comparison of compliance between non depressed and depressed patients

\begin{tabular}{|c|c|c|c|c|}
\hline Morisky Scale & No depression & Depression & P value & Total \\
\hline High Adherence & $256(54 \%)$ & $287(45 \%)$ & 0.001 & 543 \\
\hline Medium Adherence & $82(25.5 \%)$ & $241(74.4 \%)$ & 0.000 & 323 \\
\hline Low Adherence & $55(34.3 \%)$ & $105(65.5 \%)$ & 0.000 & 160 \\
\hline Total & 393 & 633 & & 1026 \\
\hline
\end{tabular}

\section{$\square$ DISCUSSION}

This study shows that frequency of hypertension is higher in depressed patients. Similarly, control of hypertension is poor and adherence to medications is less among depressed patients. Our study findings are in accordance with the observations by many international studies. Rabkin et $\mathrm{al}^{5}$ in their study conducted on 452 psychiatric outpatients with depression found that hypertension was three fold highly prevalent than those without depression. They suggest that there may be a significant association between depression and hypertension. Rabkin et $\mathrm{a}^{5}$ think that depression affcets pathophysiology of Hypertension, that's why hypertension prevalence is higher among depressed patients. Our study revealed linear association of hypertension with depression. Higher frequency of hypertension was noted among those severely depressed. Jokisalo et al shows that a feeling of hopelessness toward hypertension is associated with poor blood pressure control. ${ }^{11}$

Nakagawara et al found that there was increased frequency of hypertension in depressed patients with melancholic features. ${ }^{12}$ A prospective study found more cardiovascular diseases and hypertension in depressed patients than the other psychiatric patients. ${ }^{13}$

Kario $\mathrm{K}$ et al observed that depressive symptoms were associated with higher systolic blood pressure (SBP). ${ }^{14}$ Another study found that self-reported depression was associated with higher systolic blood pressure and diastolic blood pressure. ${ }^{15}$ These studies support the present study finding that mean systolic blood pressure and diastolic blood pressure is higher in patients with depression. Reasons of high SBP and DBP among depressed patients may be because of release of neurotransmitters such ad adrenaline and non-adrenaline. Secondly depressed patients also show less interest towards medication, rendering them vulnerable to uncontrolled Blood Pressure. Our study finding is endorsed by study by Kim HK et a ${ }^{16}$ who observed that depressed patients have low adherence level to medications and usually depression among hypertensive patients is not addressed. Consequently, such patients, by not taking their mental health into consideration, develop uncontrolled hypertension and its complications. ${ }^{16,17}$

The results of the study suggest that depression is a common factor that contributes to poor control of blood pressure in such patients. Screening depression in hypertensive patients is a simple and more effective way that may improve compliance and control of Hypertension and should be mandatory for all hypertensive patients. However, it is a single center study in which patients presenting to Lady Reading Hospital, Department of Cardiology were enrolled only. Secondly, de- pression diagnosis and compliance to medications were done on the basis of patients self-reported proforma. Recall bias in such studies, on the part of patients cannot be excluded. A large and multicenter study will be needed to generate cause and effect hypothesis among depression and hypertension.

\section{CONCLUSION}

The study concluded that hypertension was more prevalent among depressed patients. The mean systolic and diastolic blood pressure was also found to be high in depressed patients as compared to non-depressed patients. In compliance to anti-hypertensives, depressed patients had lesser compliance than the other group patients.

\section{REFERENCES}

1. National Library of Medicine- PubMed Health. Depression. www.ncbi.nlm.nih. gov/pubmedhealth/PMHT0024768/ (2017, cited 12 April 2017].

2. Waraich P, Goldner EM, Somers JM, et al. Prevalence and incidence studies of mood disorders: a systematic review of the literature. Can J Psychiatry. 2011; 49:124-38.

3. Kearney PM, Whelton M, Reynolds K, Muntner P, Whelton PK. Global Burden of hypertension: analysis of worldwide data. Lancet. 2015; 365(9455):21723.

4. Forouzanfar $\mathrm{MH}$, Alexander L, Anderson $\mathrm{HR}$, et al. Risk Factors Collaborators. Global, regional and national comparative risk assessment of 79 behavioral, environmental and occupational and metabolic risks or clusters of risks in 188 countries, 1990-2013: a systematic analysis for the Global Burden of disease study 2013. Lancet. 2015; 386(10010):2287-323

5. Rabkin J, Charles E, Kass F. Hypertension and DSM-III: Depression in psychiatric outpatients. Am J Psychiatry. 
2014;140(8):1072-4.

6. Adamis D, Ball C. Physical morbidity in elderly psychiatric inpatients: Prevalence and possible relations between the major mental disorders and physical illness. Int J Geriatr Psychiatry. 2015;15(3):248-53.

7. Thomas J, Jones G, Scarinci I, Brantley $P$. A descriptive and comparative study of the prevalence of depressive and anxiety disorders in low-income adults with type 2 diabetes and other chronic illnesses. Diabetes Care. 2016; 26:2311-7.

8. Meng L, Chen D, Yang Y, Zheng Y, Hui R. Depression increases the risk of hypertension incidence: A meta-analysis of prospective cohort studies. J Hypertension. 2012; 30:842-51.

9. O'Connor CM, Gurbel PA, Serebruany VL. Depression as a risk factor for cardiovascular and cerebrovascular disease: Emerging data and clinical perspectives. Am Heart J. 2012; 140: 63-9.

10. Zhanzhan Li, Yanyan Li, Yingyum Hu. Prevalence of Hypertension in depression. Med. 2015; 94(3): e1317.

11. Jokisalo E, Enlund H, Halonen $P$, Takala J, Kumpusalo E. Factors related to poor control of blood pressure with antihypertensive drug therapy. Blood Press. 2011; 12:49-55.

12. Nakagawara M, Witzke W, Matussek N. Hypertension in depression. Psychol Res. 2013; 21(1):85-6.

13. Kario K, Schwartz JE, Davidson KW, Pickering TG. Gender differences in associations of diurnal blood pressure variation, awake physical activity and sleep quality with negative affect. Hypertension. 2001; 38(5):997-1002.

14. Shinagawa M, Otsuka K, Murakami S, Kubo Y, Cornelissen G, Matsubayashi $\mathrm{K}$, et al. Seven-day (24 h) ambulatory BP monitoring, self-reported depression and quality of life scores. Blood Press Monit. 2002; 7(1):69-76.

15. Jonas BS, Franks $P$, Ingram DD. Are symptoms of anxiety and depression risk factors for hypertension? Longitudinal evidence from the National Health and Nutrition Examination Survey I Epidemiologic Follow-up Study. Arch Fam Med. 1997; 6(1):43-9.

16. Kim HK, Park JH. Differences in adherence to antihypertensive medication regimens according to psychiatric diagnosis: results of a Korean population-based study. Psychosom Med. 2010; 72(1): 80-87.

17. Etienne M, Hossain M, Redfield R, Stafford K, Amoroso A. Indicators of adherence to antiretroviral therapy treatment among HIV/AIDS patients in 5 African countries. J Int Assoc Provid AIDS Care. 2010; 9:98.

\section{Author's Contribution}

MAl conceptualized and designed the study, collection of data with analysis, manuscript writing and critical analysis. AK conceptualized and designed of the study, collection of data with analysis, and manuscript writing. SS conceptualized and critical analysis. MK designed the study and analyzed the data. AG conceptualized and designed the study, data with analysis and manuscript writing. Authors agree to be accountable for all aspects of the work in ensuring that questions related to the accuracy or integrity of any part of the work are appropriately investigated and resolved

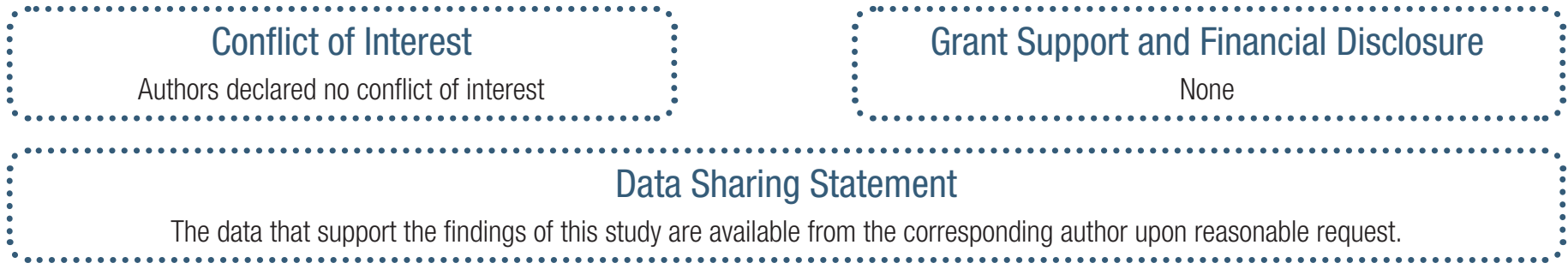

